Electronic Supporting Information for

\title{
DETERMINATION OF ENROFLOXACIN IN \\ BOVINE MILK BY A NOVEL SINGLE-STRANDED \\ DNA APTAMER CHEMILUMINESCENT ENZYME \\ IMMUNOASSAY
}

Hengjia Ni ${ }^{\mathrm{a}}$; Xinghua Ding ${ }^{\mathrm{b}}$; Tiejun Mi ${ }^{\mathrm{a}}$; Zhanhui Wang ${ }^{\mathrm{a}}$ and Suxia Zhanga*

* Correspondence Author: Suxia Zhang, Department of Pharmacology and

Toxicology, College of Veterinary Medicine, China Agricultural University, Yuan

Ming Yuan West Road No. 2, Beijing 100193, China

E-mail: suxia@cau.edu.cn

a Department of Pharmacology and Toxicology, College of Veterinary Medicine,

China Agricultural University, Yuan Ming Yuan West Road NO.2, Beijing 100193,

China

b State Key Laboratory of Proteomics and Department of Neurobiology, Department

of Neurobiology, Institute of Basic Medical Sciences, Beijing 100850, China 
Table of Contents

I. Materials and Methods
A. Apparatus and reagents
B. Immobilization of enrofloxacin onto magnetic beads
C. Preparation of enrofloxacin-spacer-HRP conjugate
D. Preparation of streptavidin-coated microplate

II. Supporting Results
A. The evaluation of the coupling between enrofloxacin and magnetic beads
B. Identification of enrofloxacin-spacer-HRP conjugates
C. The capacity of the streptavidin-coated plate.

III. Reference Cited 


\section{Materials and Methods}

\section{A. Apparatus and Reagents}

$\mathrm{N}$, N-diisopropylethylamine, bromo-tris-pyrrolidinophosphonium hexafluorophosphate, 1-[bis (dimethylamino) methylene]-1h-1, 2, 3-triazolo [4, 5-b] pyridinium 3-oxid hexafluorophosphate, 1-hydroxybenzotrizole, and 1, 6-hexanediamine were purchased from Aladdin Reagent (Shanghai, China). N, N-dimethylformamide (DMF) was purchased from J \& K Scientific (Beijing, China). Bovine serum albumin (BSA) and streptavidin were obtained from Promega (Madison, USA). EZ-Link Plus Activated Peroxidase Kit was obtained from Thermo Fisher Scientific (San Jose, Canada). Dynabeads M-270 Amine was purchased from Invitrogen. The monoclonal antibodies against enrofloxacin (E5B7E8) and chloramphenicol-HRP were previously prepared in our laboratory (Mi et al. 2013; Wang et al. 2007; Tao, Wang et al. 2013). Other reagents (analytically pure) were supplied by Beijing Reagent (Beijing, China).

The following buffers were used: blocking solution, 0.01 M sodium phophate-buffered saline (PBS) with $0.5 \%$ casein, containing $1 \%$ bovine serum albumin (BSA) and 0.1\% Proclin-300, $\mathrm{pH} 7.4$; saturation solution, $50 \mathrm{mM}$ Tris- $\mathrm{HCl}$, pH 7.0; $150 \mathrm{mM} \mathrm{NaCl} ; 0.05 \% \mathrm{NaN}_{3} ; 1 \%$ BSA and 6\% D-sorbitol; coating buffer, 100 $\mathrm{mM} \mathrm{Na} 2 \mathrm{HPO}_{4} / 50 \mathrm{mM}$, citric acid, $\mathrm{pH} 5.0$; blocking solution, $0.01 \mathrm{M}$ sodium phosphate-buffered saline (PBS) with $0.5 \%$ casein, containing $1 \%$ bovine serum albumin (BSA) and 0.1\% Proclin-300, pH 7.4; and washing buffer, sodium phosphate-buffered saline, $0.05 \%$ Tween 20 . 


\section{B. Immobilization of Antibiotics on Magnetic Beads}

Dynabeads M-270 Amine were used as the carrier for the semiautomatic selection procedure. $1.5 \mu \mathrm{mol}$ bromo-tris-pyrrolidinophosphonium hexafluorophosphate, $2 \mu \mathrm{mol}$ 1-hydroxybenzotrizole, $3 \mu \mathrm{mol} \mathrm{N}, \mathrm{N}$-diisopropylethylamine and $1 \mu \mathrm{mol}$ enrofloxacin were added into dry $\mathrm{N}, \mathrm{N}$-dimethylformamide and activated for $2 \mathrm{~h}$ at room temperature. Then $100 \mu \mathrm{L}$ (about $2 \times 10^{8}$, or $0.3 \mu \mathrm{mol}$ ) magnetic beads were added to the mixture and stirred overnight (Stern et al. 2005). After the reaction was complete, the coated beads were washed with binding buffer and stored at $4{ }^{\circ} \mathrm{C}$ until use. The attachment was qualitatively verified by calculating the concentrations of enrofloxacin in the supernatant before and after attachment. The $\pi$-conjugated system made fluoroquinolones detectable by fluorescence spectroscopy, and the excitation and emission wavelength were $280 \mathrm{~nm}$ and $450 \mathrm{~nm}$ (Ramos et al. 2003). All samples were diluted into the linear dynamic range before analysis. The ofloxacin coating procedure was performed identically as for enrofloxacin.

\section{Preparation of enrofloxacin-spacer-HRP conjugate}

Before conjugation, enrofloxacin was modified to form a spacer (Lüttenberg, Sondermann and Scherkenbeck 2012). Briefly, 5 mg enrofloxacin, 8 mg 1- [bis (dimethylamino) methylene]-1h-1, 2, 3-triazolo [4, 5-b] pyridinium 3-oxid hexafluorophosphate and 7.4 $\mu \mathrm{L} \mathrm{N}, \mathrm{N}$-diisopropylethylamine were dissolved in $\mathrm{N}$, $\mathrm{N}$-dimethylformamide, then stirred for $2 \mathrm{~h}$ at $4{ }^{\circ} \mathrm{C}$. Then $10 \mathrm{mg}$ 1,6-hexanediamine (spacer, dissolved in methanol) were added into the reaction mixture and stirred at room temperature overnight. The enrofloxacin-spacer was separated by thin layer 
chromatography (TLC) using $\mathrm{CH}_{2} \mathrm{Cl}_{2} /$ methanol $(2: 1, \mathrm{v} / \mathrm{v})$ as the eluent. The luminous band obtained at $\mathrm{R}_{\mathrm{f}}=0.25$ for the enrofloxacin-spacer was scraped from the plate and extracted with methanol. The methanol was evaporated under a stream of nitrogen. The connection between enrofloxacin-spacer and HRP was performed using the EZ-Link Plus Activated Peroxidase Kit. The conjugate was confirmed by using enrofloxacin antibody (1:200) coated microplates. Briefly, $100 \mu \mathrm{L}$ of enrofloxacin antibodies were added into each well and incubated at $4^{\circ} \mathrm{C}$ overnight. The plates were washed three times with sodium phosphate-buffered saline, and treated with blocking solution (200 $\mu \mathrm{L} /$ well) for $1.5 \mathrm{~h}$ at $37^{\circ} \mathrm{C}$. After rinsing five times with washing buffer, a series of concentrations of enrofloxacin-spacer-HRP was added into the prepared microwells. After incubation for $30 \mathrm{~min}$ at $25^{\circ} \mathrm{C}$, the plates were washed 5 times. Then $50 \mu \mathrm{L}$ of the chemiluminescence substrate solution were added to each well, and the luminescence was measured after incubation for $5 \mathrm{~min}$. Chloramphenicol-HRP was used as a control to verify the reliability of the confirmation.

\section{Preparation of Streptavidin-Coated Microplate}

The white microplates were coated with streptavidin. The coating procedure was performed as previously reported (Välimaa et al. 2002). Briefly, $2 \mathrm{mg} / \mathrm{mL}$ streptavidin were added into $1 \%$ glutaraldehyde, and the mixture was incubated for $2 \mathrm{~h}$ at $4{ }^{\circ} \mathrm{C}$. After removing excess glutaraldehyde, the treated streptavidin was then diluted in the coating buffer $(5 \mu \mathrm{g} / \mathrm{mL}) .200 \mu \mathrm{L}$ of the coating solution was dispensed into each well and incubated overnight at $35^{\circ} \mathrm{C}$. The plates were washed, and saturation solution $(250$ $\mu \mathrm{L}$ ) was added per well. The plates were saturated overnight at $25^{\circ} \mathrm{C}$. The saturation 
solution was aspirated and the plates were dried $\left(35^{\circ} \mathrm{C}\right.$, relative humidity $\left.5 \%\right)$ for $2 \mathrm{~h}$. Finally, the plates were packed with adsorbent and stored dry at $4^{\circ} \mathrm{C}$. The capacities of the coated wells were determined. A series of concentrations of biotin-labeled No.17 $(100 \mu \mathrm{L})$ were added into three replicate wells. The plates were incubated for $30 \mathrm{~min}$ at $25^{\circ} \mathrm{C}$, and the supernatant of each well was homogenized by vigorous pipetting before collected. The optical densities (OD) of the supernatant were determined at $260 \mathrm{~nm}$ $\left(\mathrm{OD}_{260}\right)$ before and after the incubation. The changes in $\mathrm{OD}_{260}$ were used to evaluate the binding capacity of the streptavidin-coated microplate.

\section{Supporting Results}
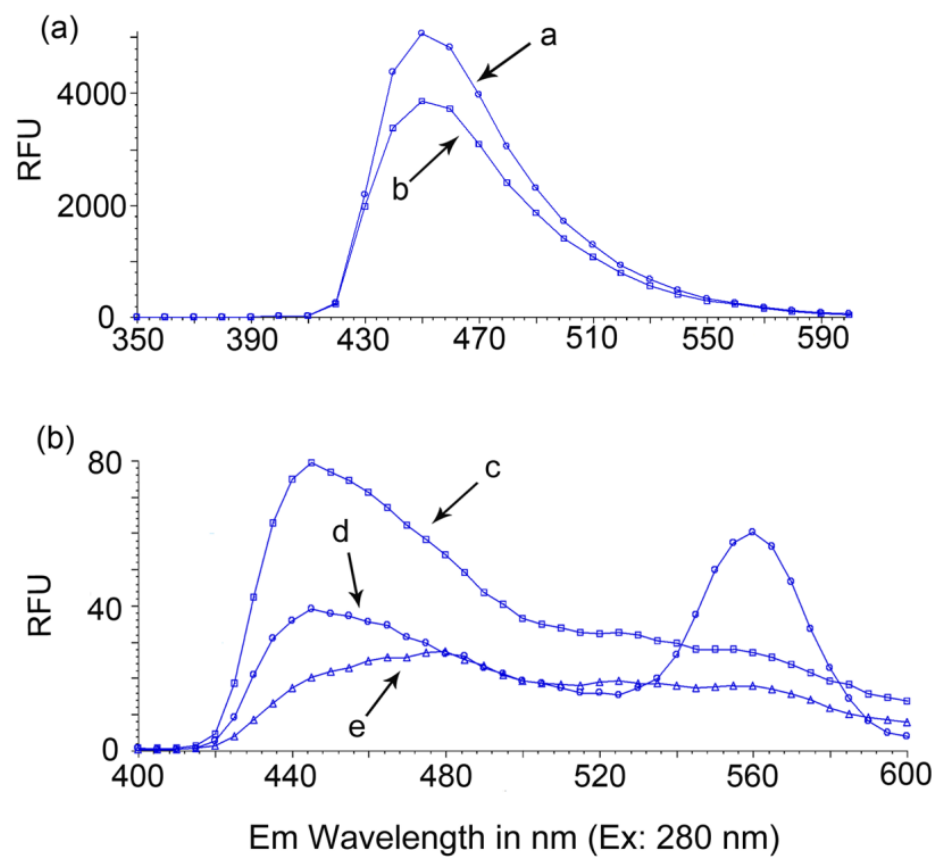

Figure S1. Evaluation of the coupling between enrofloxacin and magnetic beads:

(a) Quantitative analysis of the reaction between enrofloxacin and magnetic beads; line a represents the initial pool of enrofloxacin; line b represents the pool of unbound enrofloxacin after coating. (b) Qualitative analysis of the reaction between enrofloxacin 
and magnetic beads; line c represents enrofloxacin-coated magnetic beads; line $\mathrm{d}$ represents magnetic beads in the buffer; and line e represents the uncoated magnetic beads.

To evaluate the result of immobilization, the concentration of the bound enrofloxacin was calculated by the difference in initial and unbound concentration. Figure S1a showed that the peak of unbound enrofloxacin at $450 \mathrm{~nm}$ was much lower than that of the initial one. Moreover, the decrease of enrofloxacin in the reaction buffer was the amount of enrofloxacin that had been attached on the magnetic beads. According to Lambert-Beer's law (Ricci, Ditzler, and Nestor 1994), there is a linear relationship between the relative fluorescent intensity (RFU) and concentration of enrofloxacin under the same path length. Therefore, the coupling ratio $(\mathrm{R})$ can be calculated as:

$\mathrm{R}=\left(\mathrm{N}_{\text {before reaction }}-\mathrm{N}_{\text {before reaction }} \times \mathrm{S}_{\text {after reaction }} / \mathrm{S}_{\text {before reaction }}\right) / 0.3$

In this equation, $\mathrm{N}$ is the amount of enrofloxacin, and $\mathrm{S}$ is fluorescence signal. According to this result, it can be concluded that approximately $80 \%$ of magnetic beads had been coated by enrofloxacin. As it showed from Figure S1b, line c represented enrofloxacin-coated beads and showed a distinct peak at $450 \mathrm{~nm}$ which was the major peak of enrofloxacin. The peak of enrofloxacin-coated beads at $450 \mathrm{~nm}$ was higher than that of preservation buffer and uncoated beads. These results suggested that the beads were successfully coated with enrofloxacin. 


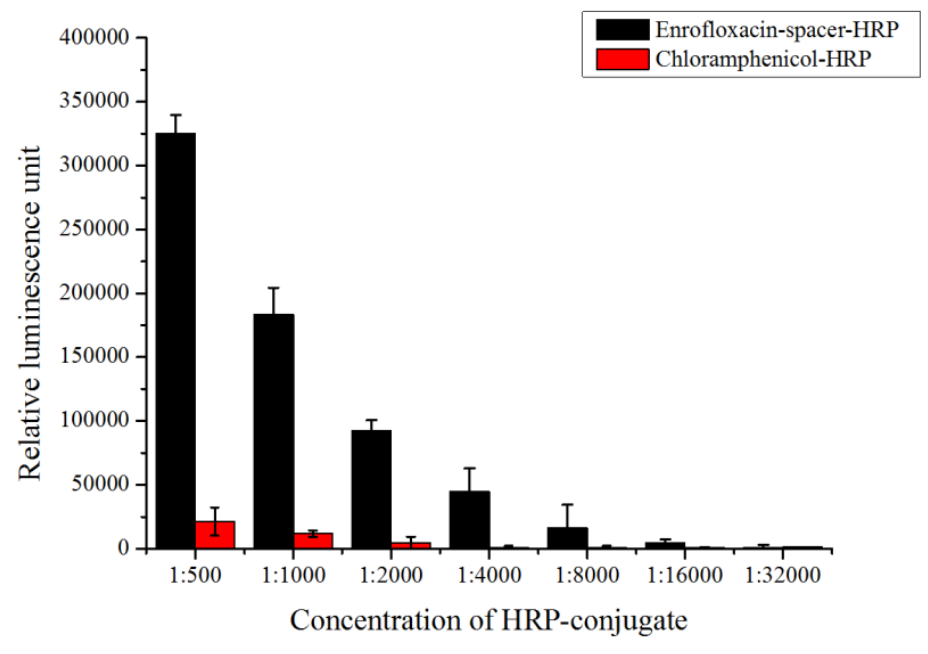

Figure S2 Identification of enrofloxacin-spacer-HRP conjugates: The synthesis of enrofloxacin-spacer-HRP was confirmed by the antibody-coated plate. Non-specific binding to the antibody-coated plate was also assessed by comparing the RLUs obtained from enrofloxacin-spacer-HRP and chloramphenicol-HRP at the same dilution. The results showed that the RLUs of enrofloxacin-spacer-HRP decreased along with an increase in the dilution factor. For chloramphenicol-HRP, only a low quantity of RLUs was measured even using dilution 1:500. These results indicated that the antibody-coated plate exhibited low non-specific binding. Furthermore, these results showed that the prepared antibody-coated microplate was able to specifically recognize the developed HRP products that had been loaded into the wells, which means enrofloxacin-spacer-HRP were synthesized successfully. 


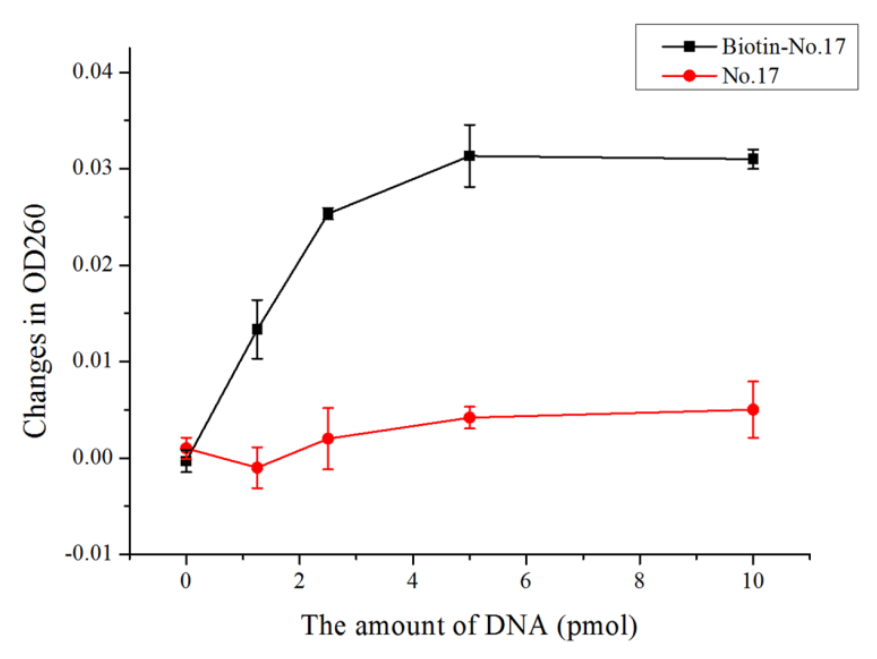

Figure S3 Capacity of the streptavidin-coated plate: The streptavidin-coated plates were prepared according to previous research, and the reported capacities of the plates to biotinylated moleculars were more than $3.6 \mathrm{pmol}$. The binding capacities of the coated wells in this study were determined by adding different concentrations of biotin-labeled number 17 and number 17 into streptavidin-coated plates. Values $>0$ indicated a combination between DNA and microplate. After incubation, biotin-labeled number 17 had more changes in $\mathrm{OD}_{260}$ than number17. Besides, the $\mathrm{OD}_{260}$ values of biotin-labeled number 17 almost reached a maximum of 0.03 when the added amount was $2.5 \mathrm{pmol}$. These results indicated that the binding capacity of the coated well was no more than 2.5 pmol.

\section{References Cited}

Lüttenberg, S., F. Sondermann, and J. Scherkenbeck. 2012. Anthelmintic PF1022A: stepwise solid-phase synthesis of a cyclodepsipeptide containing $\mathrm{N}$-methyl amino acids. Tetrahedron. 68: 2068-2073.

Mi, T., Z. Wang, S. A. Eremin, J. Shen, and S. Zhang. 2013. Simultaneous Determination of Multiple (Fluoro)quinolone Antibiotics in Food Samples by a One-Step Fluorescence Polarization Immunoassay. J. Agric. Food Chem. 61: 
9347-9355.

Ramos, M., A. Aranda, E. Garcia, T. Reuvers, and H. Hooghuis. 2003. Simple and sensitive determination of five quinolones in food by liquid chromatography with fluorescence detection. J. Chromatogr. B. 789: 373-381.

Ricci, R. W., M. Ditzler, and L. P. Nestor. 1994. Discovering the Beer-Lambert Law. J. Chem. Educ. 71: 983.

Stern, E., G. G. Muccioli, R. Millet, J. F. Goossens, A. Farce, P. Chavatte, J. H. Poupaert, D. M. Lambert, P. Depreux, and J. P. Hénichart. 2005. Novel 4-Oxo-1,4-dihydroquinoline-3-carboxamide Derivatives as New CB2 Cannabinoid Receptors Agonists: Synthesis, Pharmacological Properties and Molecular Modeling. J. Med. Chem. 49: 70-79.

Tao, X., W. Wang, Z. Wang, X. Cao, J. Zhu, L. Niu, X. Wu, H. Jiang, and J. Shen. 2013. Development of a highly sensitive chemiluminescence enzyme immunoassay using enhanced luminol as substrate. Luminescence.

Välimaa, L., K. Pettersson, M. Vehniäinen, M. Karp, and T. Lövgren. 2002. A High-Capacity Streptavidin-Coated Microtitration Plate. Bioconjugate Chem. 14: 103-111.

Wang, Z., Y. Zhu, S. Ding, F. He, R. C. Beier, J. Li, H. Jiang, C. Feng, Y. Wan, S. Zhang, Z. Kai, X. Yang, and J. Shen. 2007. Development of a Monoclonal Antibody-Based Broad-Specificity ELISA for Fluoroquinolone Antibiotics in Foods and Molecular Modeling Studies of Cross-Reactive Compounds. Anal. Chem. 79: 4471-4483. 